\title{
Control de humedad y consumo de agua en un invernadero
}

\author{
Secundino Marrero Ramírez \\ ORCID: https://orcid.org/0000-0001-5161-545X \\ secundino.marrero@utc.edu.ec \\ Universidad Técnica de Cotopaxi \\ Latacunga, Ecuador \\ Marco Anibal León Segovia \\ ORCID: https://orcid.org/0000-0002-5622-1471 \\ correo@institucion.com \\ Universidad Técnica de Cotopaxi \\ Latacunga, Ecuador
}

\author{
Iliana González Palau \\ ORCID: https://orcid.org/0000-0001-2057-7472 \\ iliana.gonzalez@utc.edu.ec \\ Universidad Técnica de Cotopaxi \\ Latacunga, Ecuador \\ Rommel Eusebio Suarez Vinueza \\ ORCID: https://orcid.org/0000-0002-7545-6286 \\ correo@institucion.com \\ Universidad Técnica de Cotopaxi \\ Latacunga, Ecuador
}

Recibido (16/04/21), Aceptado (10/05/21)

Resumen: La investigación se realizó en un el invernadero experimental inacral para el control de humedad y consumo de agua, con el uso de sensores de temperatura, humedad, nivel y un PLC a través de un sistema Scada. Se pudo establecer el control manual y automático de la humedad en los rangos requeridos y con una electroválvula se controla el llenado del tanque con los niveles mínimo y máximo programados. En la interfaz gráfica (HMI) se controla y monitorea de forma remota el gasto de agua y el comportamiento de la humedad. También es posible conocer el consumo de agua diario, semanal y mensual, así como el nivel y volumen del agua existente en el tanque. El sistema facilitó el trabajo en el invernadero, mejoró la calidad del cultivo y permitió el seguimiento del gasto de agua por plantas, la dosificación correcta y el uso racional de este recurso.

Palabras Clave: Control, Sensores, Scada, HMI.

\section{Control of humidity and water consumption in a greenhouse}

Abstract: The research was carried out in an experimental inacral greenhouse for the control of humidity and water consumption, with the use of temperature, humidity, level sensors and a PLC through a Scada system. It was possible to establish manual and automatic humidity control in the required ranges and with a solenoid valve the tank filling is controlled with the minimum and maximum levels programmed. With the graphical interface (HMI) you can remotely control and monitor water consumption and humidity behavior. It is also possible to know the daily, weekly and monthly water consumption, as well as the level and volume of the water in the tank. The system contributed to the humanization of the work, improved the quality of the crop and facilitated the monitoring of water consumption by plants, the correct dosage and the rational use of this resource.

Keywords: Control, Sensors, Scada, HMI 


\section{Introducción}

En los invernaderos se requieren condiciones artificiales favorables y dentro de ellos se consigue un aislamiento térmico y de agentes contaminantes de los cultivos. Sin embargo, es necesario que estos cuenten con sistemas que permitan controlar y modificar las condiciones del microclima generado. En trabajos como [1], [2], [3] se definen las variables a controlar en un invernadero, como temperatura del aire, humedad relativa, concentración de $\mathrm{CO} 2$ y la radiación solar. Por ello, se utilizan diferentes técnicas para el control de estos parámetros a través de la instalación de calefacción, ventilación, sistema de humidificación, dosificación de $\mathrm{CO} 2$, el uso de pantallas térmicas y la iluminación artificial. La ventilación se utiliza también para prevenir excesiva humedad ambiente, debido a la transpiración del cultivo. Este control se realiza con el objeto de evitar enfermedades, ya que los excesos de humedad tienen lugar en los periodos fríos, y su efecto es más pronunciado durante los cambios de la noche al día. Además, la humedad es importante para la fotosíntesis, pues si la planta pierde demasiada agua, las estomas se cerrarán y esto provocará que la fotosíntesis se frene. Cuando esto ocurre, no se podrá absorber más $\mathrm{CO} 2$, y esto resulta necesario para mantener en marcha la fotosíntesis, entonces para tener las estomas abiertas es necesario reducir la evaporación de la planta cuando hay más radiación. Si se mantiene un nivel alto de humedad en el invernadero, la evaporación se reducirá.

La temperatura del invernadero también puede reducirse introduciendo humedad, esto provoca que la planta tenga que enfriarse menos a través de la evaporación. Por otro lado, el cultivo puede humedecerse ligeramente, y esto hace que el agua evaporada pueda enfriar el cultivo o la temperatura al interior del invernadero. En los trabajos [4], [5]se propone un modelo neuro - difuso para combinar ventajas de las redes neuronales y de los sistemas difusos que muestran un desempeño superior, especialmente en el caso de sistemas complejos y no-lineales como los presentes en el ambiente del invernadero, para predecir, controlar el comportamiento de la temperatura y la humedad relativa del aire en el interior. Estos modelos de forma satisfactoria pueden predecir el comportamiento del clima, aspecto este que contribuye significativamente al desarrollo de los sistemas de control de humedad y consumo de agua [6]
Desde el punto de vista de control, existen al menos dos umbrales de humedad relativa, uno para la noche y otro para el día, en consonancia con los puntos de consigna establecidos para la temperatura, asignando a cada uno, la curva con los déficits de humedad admisibles máximos y mínimos. Es por ello que el trabajo se enfoca en la necesidad de tener un control preciso de la humedad y el consumo del agua, para que se incorpore al sistema ya existente, de registro de otras variables del invernadero, para así lograr el ahorro del consumo de agua y la dosificación adecuada en los diferentes cultivos. En el trabajo [7] también se hace referencia a la interacción humedad-temperatura en el invernadero, misma que es evaluada a través de un modelo climático que estima la evolución horaria de la temperatura del aire y la humedad relativa en el interior de un invernadero con ventilación natural de tipo cenital y atendiendo al comportamiento del clima externo. Aquí se incorporan los efectos de la ventilación natural para enfriar el invernadero y como resultado de la evaluación, se obtuvo un ajuste adecuado para la estimación de la temperatura del aire y su interrelación con la humedad relativa en el interior del invernadero para lograr una buena cosecha.

En esta investigación se ha monitoreado la variable del consumo de agua, a través de la programación de un sistema Scada con interfaces gráficas, permitiendo de esta manera complementar el monitoreo de las variables de operación en el invernadero. La importancia de estas variables ha sido evaluada en trabajos como [8], [9] donde, a través de datos experimentales generados durante el ciclo de producción, se ha podido validar la incidencia de la humedad en el buen desarrollo del cultivo y se han identificado modelos para pronóstico de la producción.

Al incorporar las variables humedad y consumo de agua al monitoreo, no solo se contribuye con el ahorro de este recurso, sino que se facilita la labor del operador del invernadero, al poder este realizar un registro de datos del consumo y controlar el volumen de agua requerido por tipo de cultivo. Además, al incorporar nuevas variables en el control automático de las condiciones climatológicas, se pueden mejora las condiciones de producción y explotación del invernadero. El trabajo fue realizado en el invernadero experimental de tipo inacral para la obtención de plantines con una distribución de tres hileras como se observan en la figura 1

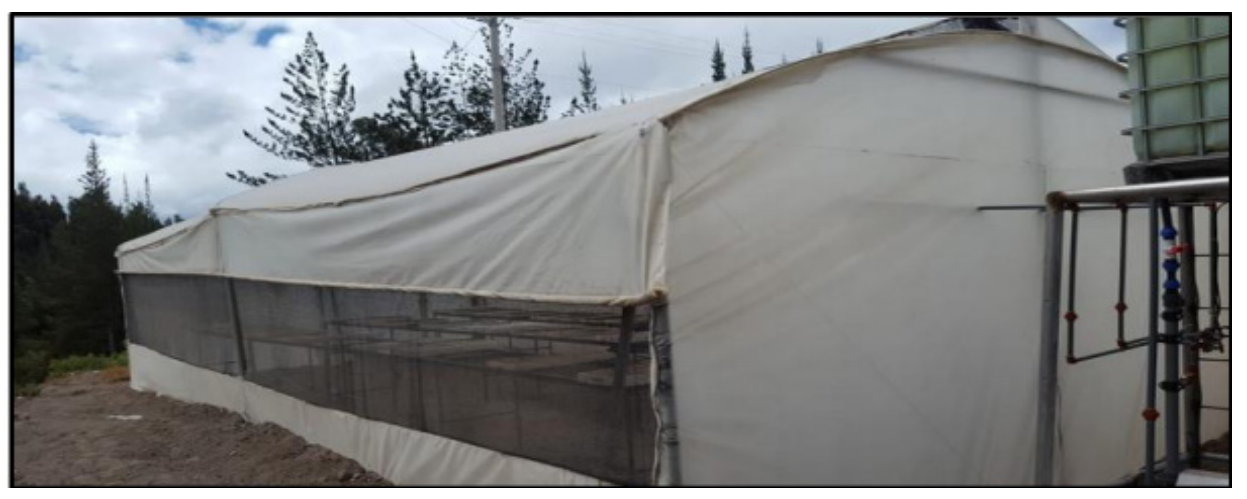

Fig. 1. Vista lateral del invernadero 
También se debe señalar, que la realización de este prototipo de sistema de monitoreo y control, permite tener a disposición del sector agrícola de la zona, una propuesta que mejora la explotación de invernaderos y que humaniza este trabajo.

\section{Desarrollo}

\section{A.Sistema Scada y control de humedad}

El sistema Scada permite la retroalimentación en tiempo real con los dispositivos de campo (sensores y actuadores) y controla las variables que han sido seleccionadas, como es el caso de la temperatura, humedad y consumo de agua [6], [7]. En la figura 2 se muestra el esquema del sistema de control realimentado, que se compone de cuatro partes fundamentales, el invernadero como objeto de control, el sistema de medida o elementos que realizan una estimación del valor de la variable a controlar (Sensor de humedad y nivel de agua del tanque), controlador (PLC, Scada) y los actuadores que son los dispositivos utilizados por los controladores para ejecutar las ordenes, que deben mantener a la variable en los límites deseados a pesar de la presencia de perturbaciones como la temperatura, radiación y humedad externa, velocidad y dirección del viento y la ocurrencia de lluvias.

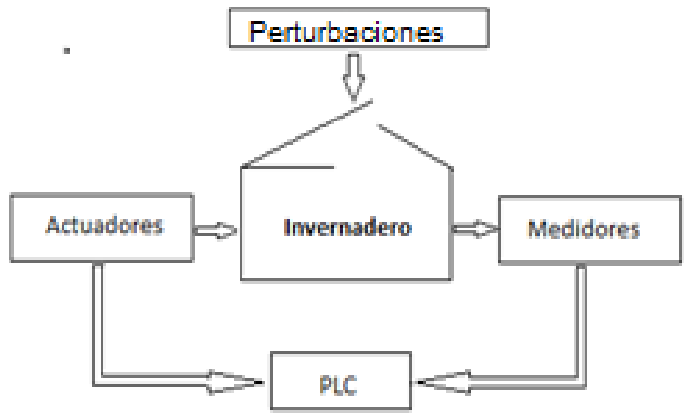

Fig. 2. Esquema de automatización

Dentro de los sistemas Scadas más utilizados en la industria, se destacan el RSView32 de Allen Bradley que es un software basado en Windows para la creación y ejecución de aplicaciones de adquisición de datos, monitoreo y aplicaciones de control. Está diseñado para operar en el ambiente de MS Windows y es compatible con contenedores OLE. También el OASYS DNA, comercializado por TELVENT, presenta un entorno que da respuestas a requerimientos relacionados con la automatización y el control de procesos industriales en tiempo real. Esta es una aplicación cliente-servidor, que permite crear un sistema óptimo y distribuido, con amplia flexibilidad para el mantenimiento y en la administración del sistema. Por otra parte, existe el WinCC, que es un sistema con HMI eficiente para la entrada de datos bajo Microsoft Windows. Este, al combinarse con el PLC para el control del proceso, permite una comunicación eficaz con el operador. Por ello fue seleccionado el WinCC para la apli- cación, atendiendo a los requerimientos y su capacidad de soportar un gran número de configuraciones, que van desde un sistema de un solo puesto hasta los sistemas redundantes distribuidos que tienen varios servidores, pasando por sistemas cliente - servidor [10], [11]. La aplicación fue desarrollada sobre la base de un PLC S7 1200 de Siemens de la serie SIMATIC, ya existente en la instalación para el control de temperatura y el consumo de energía.

En la comunicación se utiliza un canal de datos de 5Mbps de velocidad, que permite configurar equipos del tablero de control a ser monitoreados, los cuales establecen conexión mediante un switch.

\section{B.Medidores de nivel del líquido.}

La medición de nivel de líquidos para el registro indirecto del consumo de agua, suele presentar dificultades relacionadas con la medición continua, y la densidad del líquido. Para efectuar esta tarea existen instrumentos con diferentes tipos de sensores, que utilizan características eléctricas del líquido y se clasifican en: medidor conductivo, capacitivo, ultrasónico, de radiación y láser entre otros.

De esta gama de equipos se destacan las prestaciones del medidor ultrasónico en cuanto a precisión y control continuo. Aquí se emite cíclicamente un impulso acústico de alta frecuencia y corta duración que se refleja en la superficie del líquido y regresa luego de cierto tiempo, lo que se traduce en la distancia d1 y d2 como se observa en la figura 3.

El sensor para cálculo debe convertir la distancia en el nivel del líquido a través del transductor - receptor [12]. La distancia hasta el nivel de líquido se expresa por la ecuación:

$$
h=\frac{v t}{2}
$$

Dónde:

$\mathrm{h}=$ nivel del líquido

$\mathrm{v}=$ velocidad del sonido en el líquido

$\mathrm{t}=$ tiempo de tránsito del sonido

Como la distancia hacia el objeto es medida por medio del tiempo de recorrido del sonido, y no por una medición de la intensidad, los sensores ultrasónicos son insensibles al ruido de fondo

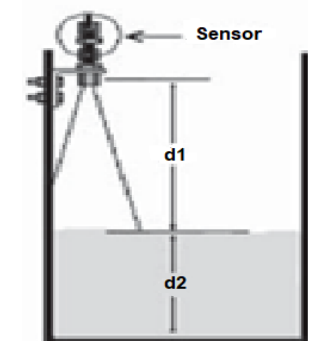

Fig. 3. Principio del medidor de nivel ultrasónico Fuente: [12] 
Prácticamente todos los materiales que reflejan el sonido son detectados y su principal dificultad se relaciona con la variación de temperatura, la presencia de burbujas o gas y la turbulencia extrema, que puede introducir error en la medición. La tabla I muestra las especificaciones técnicas del medidor sin contacto de la marca Hautu, utilizado en la medición y control del nivel de líquido que permite la transmisión de datos y la comunicación con el PLC.
La salida del medidor ultrasónico es de $4 \sim 20 \mathrm{~mA}$, donde se requiere una fuente de voltaje de 24 DC con vista a lograr el nivel mínimo establecido en el tanque de $150 \mathrm{~L}$ que asegure al menos cuatro días de operación y un máximo que se corresponde con la capacidad total del tanque de 444 L. El modelo de PLC admite una señal de entrada de voltaje, por lo que fue necesario utilizar un conversor de señal corriente a voltaje.

Tabla 1. Especificaciones técnicas del sensor de nivel Hautu.

\begin{tabular}{|l|l|}
\hline \multicolumn{2}{|c|}{ Trans misor De Nivel Ultrasónico. SERIE -21204173 } \\
\hline Rango de medición & $0-20 \mathrm{~m}$ \\
\hline Rango de precisión & $\pm 0.3 \%$ \\
\hline Modo de salida & $4 \sim 20 \mathrm{~mA}$ \\
\hline Tensión de alimentación & DC12V / DC24 \\
\hline Tiempo de respuesta & \\
\hline
\end{tabular}

Entonces para la arquitectura del sistema de control se utiliza la configuración mostrada en diagrama de bloques de la figura 4, donde se va a utilizar la medición de los sensores de humedad en las hileras del invernadero y el medidor de nivel para monitorear el volumen de agua del tanque con vista a que el PLC realice el accionamiento de la electroválvula de abasto de agua al tanque y el encendido de la bomba de riego según los requerimientos de humedad programados.

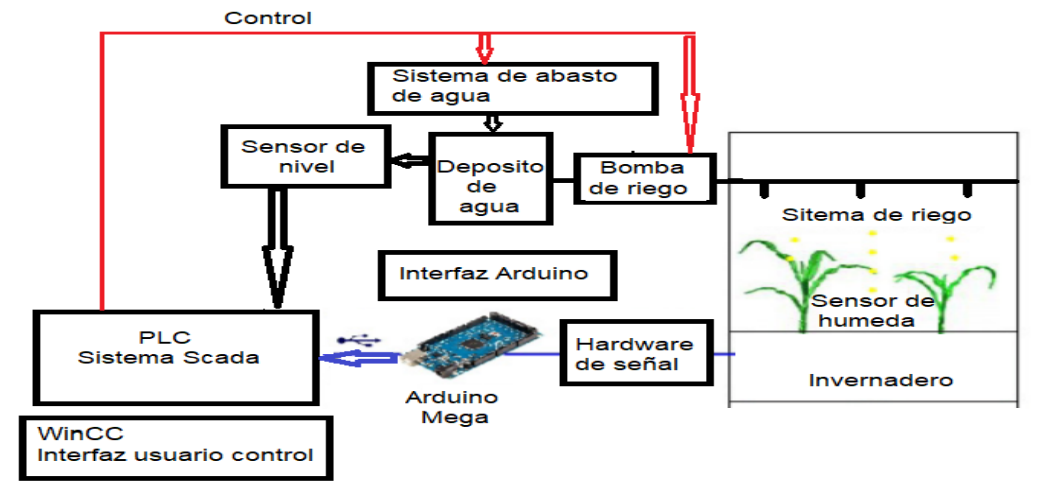

Fig. 4. Arquitectura del sistema de hardware y software del invernadero

\section{III.Resultados}

La configuración inicial propuesta del sistema Scada se muestra en la figura 5, donde se indican los equipos monitoreados y los datos de las variables climatológicas temperatura y humedad a tener en cuenta. Mientras que el algoritmo del diagrama del control de humedad se muestra en la figura 6 , aquí el control se puede realizar de manera manual o automática y para ello se introducirá el porcentaje de humedad en rangos de $30 \%$ a $60 \%$ en dependencia del tipo de cultivo y atendiendo al tipo de suelo, sí el \% es menor al valor introducido, se encenderá la bomba para el riego en las hileras requeridas según los requerimientos del cultivo plantado. 


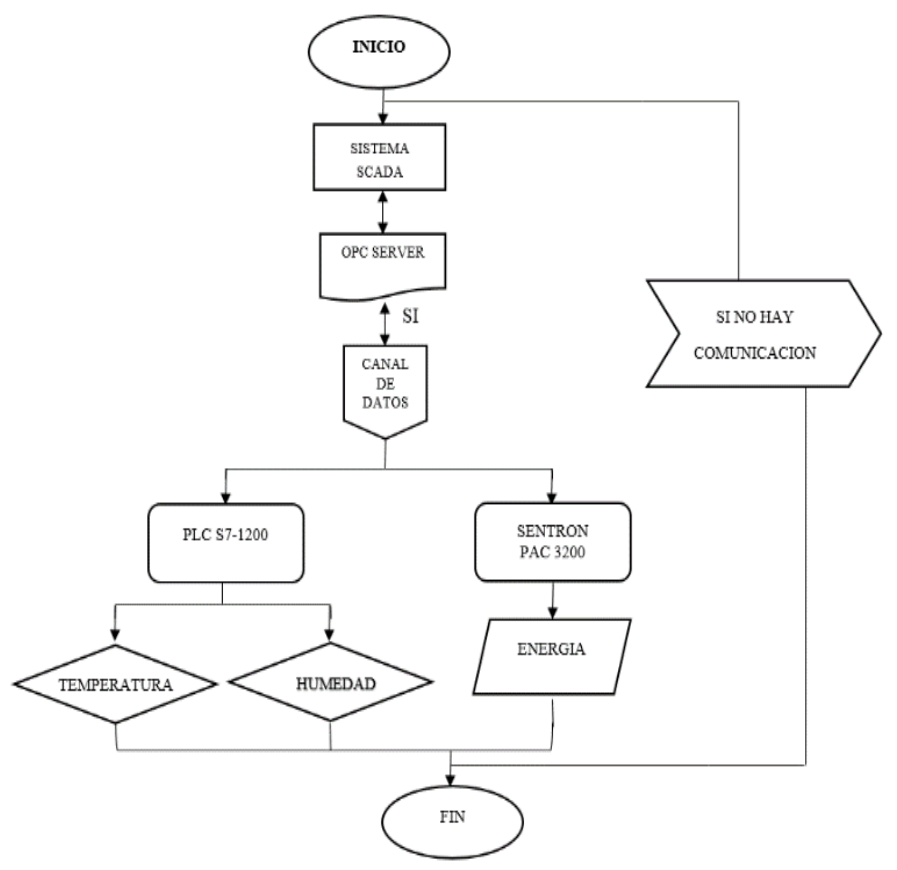

Fig. 5. Configuración inicial del Scada

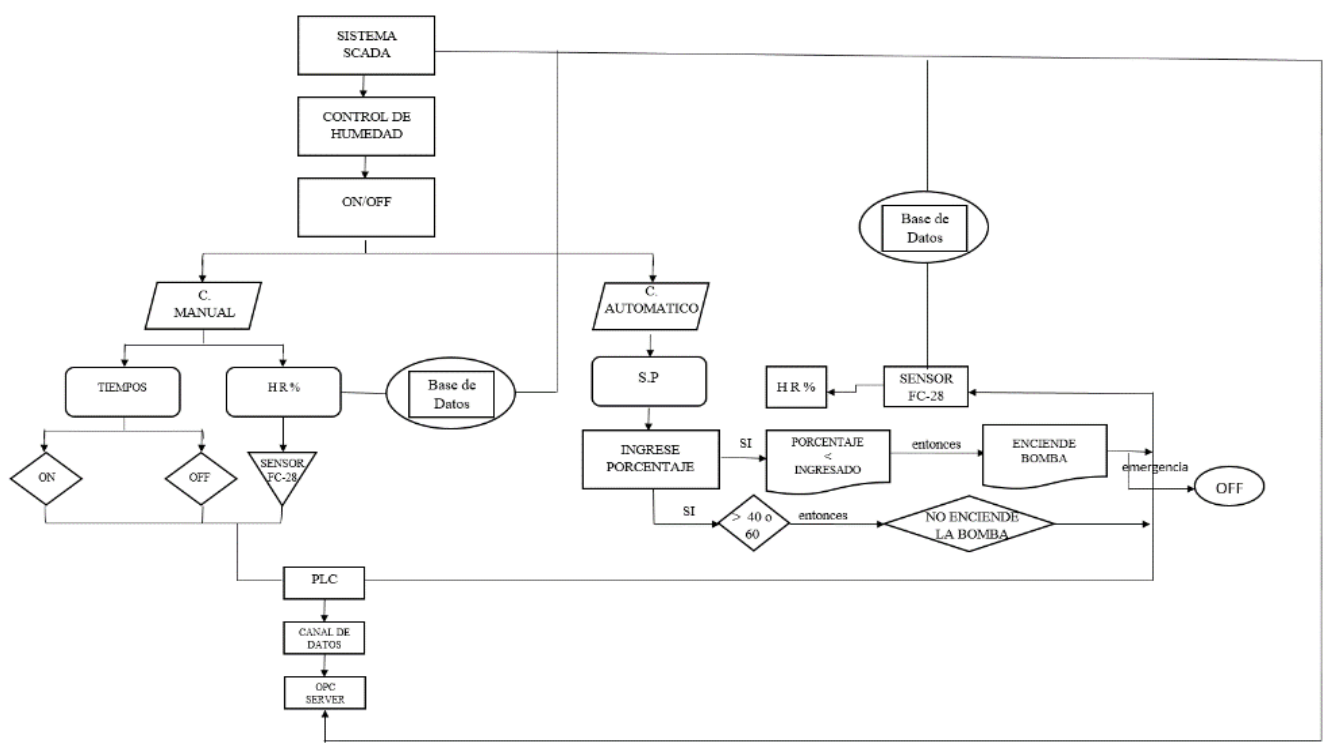

Fig. 6. Configuración del control de humedad

Cuando la operación del sistema es manual, se debe introducir el tiempo de riego en minutos para la operación de la bomba a través de la pantalla HMI que se muestra en la figura 7 , mientras que el régimen automático permite la opción de variar el nivel de humedad (H. R. \%) de $0 \%$ a $100 \%$ acorde a las necesidades del operador (S. P. \%). Se utilizó el higró- metro de suelo FC-28 de la figura 8 que es un sensor que mide resistencia con ajuste de sensibilidad y tiempo de respuesta en el orden de los segundos, el ajuste de sensibilidad se realiza por el potenciómetro del circuito acondicionador de señal con el comparador LM393 para salidas digital y analógica (entre 0 y 1023). 


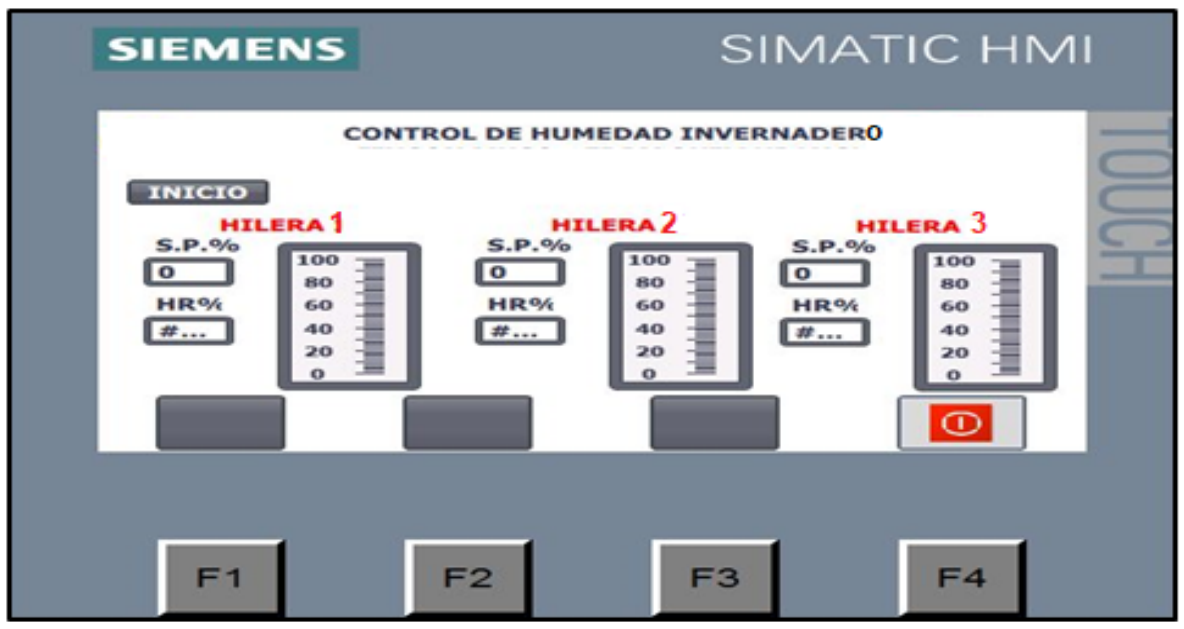

Fig. 7. Pantalla HMI del control de riego por hileras

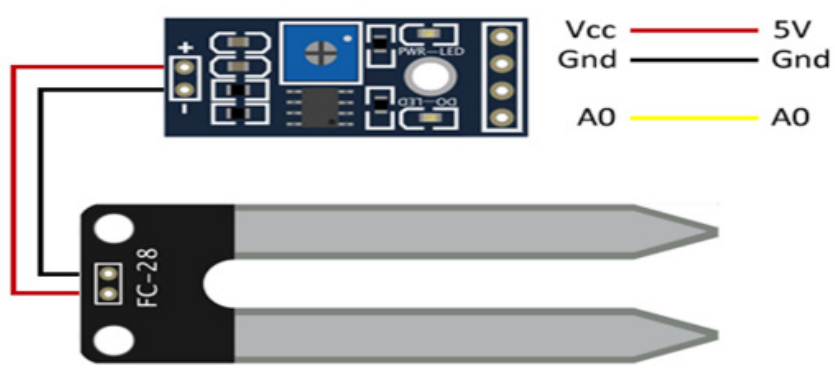

Fig. 8. Higrómetro FC-28 y placa de conexión

Para lograr una mayor precisión de la medición, se calibraron los sensores con el medidor digital HM 445 de precisión $\pm 5 \%$, considerando el valor de 100 cuando la humedad es del $60 \%$ y se obtuvo una precisión que es aceptable para activar el sistema de riego como se sugiere en [13] y [14], donde se considera que el \% de humedad relativa se debe establecer atendiendo al tipo de suelo y cultivo. También se utiliza una tarjeta Mega Arduino con un circuito para llevar la señal de los sensores al PLC, cuya placa fue diseñada en Proteus para colocar 8 sensores de humedad, alimentados con una fuente de corriente continua y el circuito impreso obtenido para colocar las tarjetas de ajuste y calibración de los sensores en cada una de las hileras del invernadero se puede observar en la figura 9.

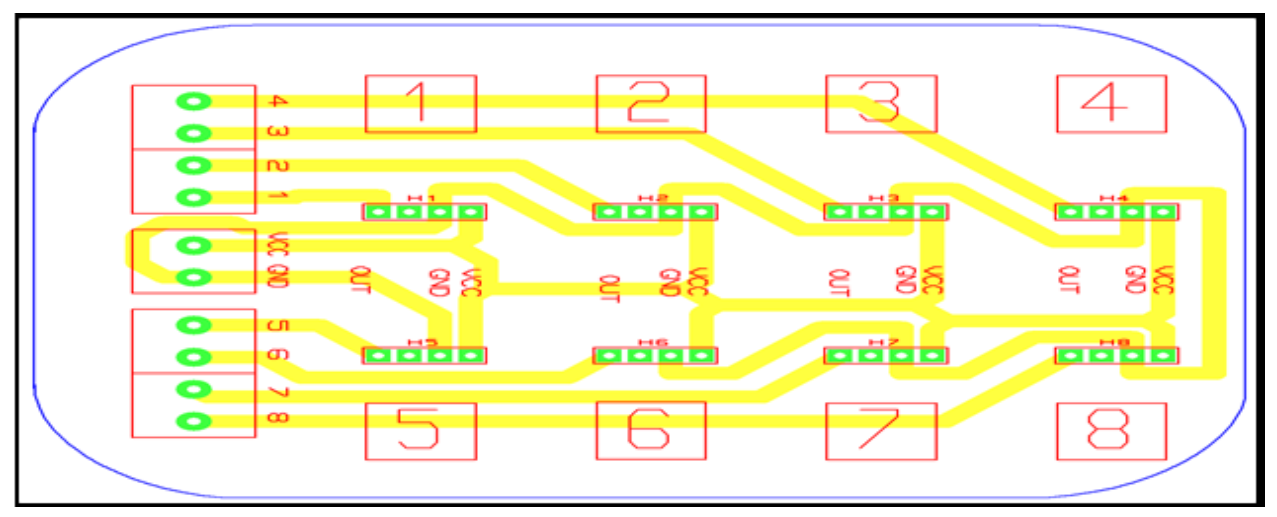

Fig. 9. Placa de acondicionamiento de señal de los sensores de humedad 
El esquema de conexión de los sensores para el riego de las tres hileras del invernadero es mostrado en la Figura 10, esto facilita que se pueda realizar de forma independiente el riego por hileras con vista a satisfacer las necesidades de humedad de diferentes cultivos localizados en las mismas. Ade- más, de esta forma si no se han plantado semillas en todas las hileras, entonces se puede seleccionar la operación de aquella que está sembrada para no realizar un gasto innecesario de agua.

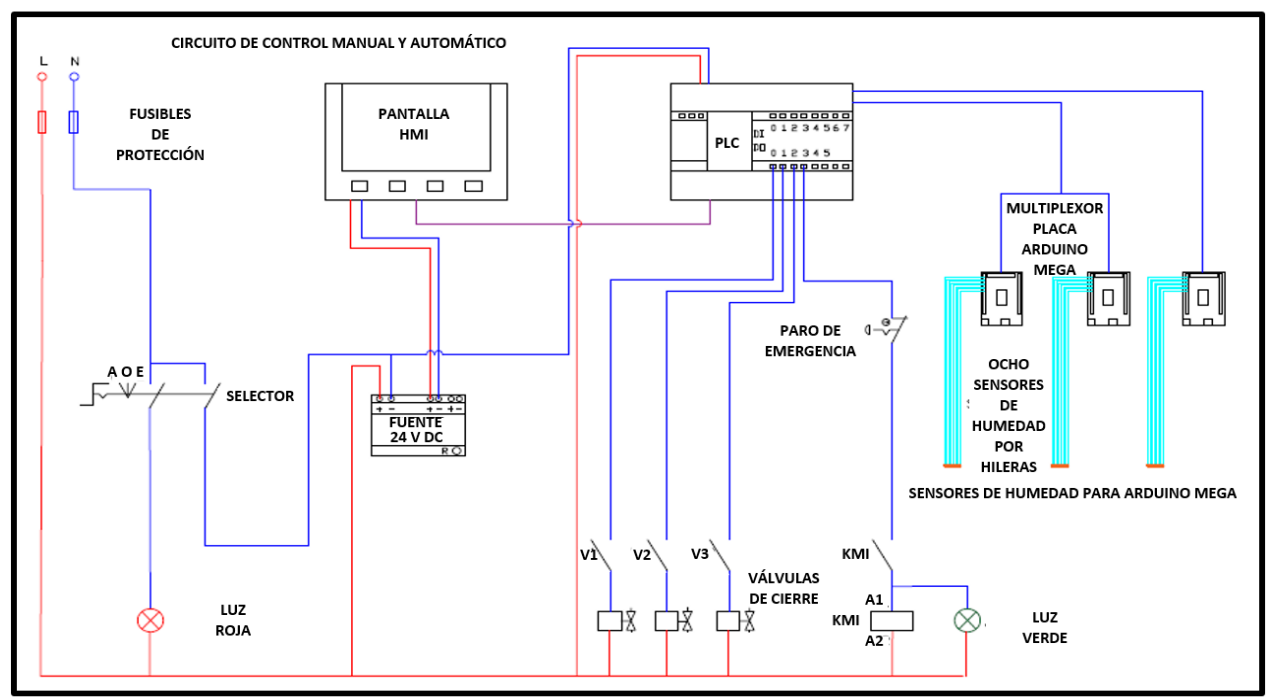

Fig. 10. Esquema eléctrico de conexión de sensores de humedad

Al tener que incorporar el monitoreo del consumo de agua, se propone el algoritmo general de control del invernadero de la figura 11 con el esquema de control para el consumo de agua que se muestra en la figura 12, donde es utilizada una electroválvula del tipo diafragma, que puede entregar entre $38 \mathrm{~L} / \mathrm{min}$ a $240 \mathrm{~L} / \mathrm{min}$ para garantizar el llenado del tanque, una vez que el sistema alcanza el mínimo o máximo prestablecido. Con vista a garantizar la seguridad en la explotación del sistema se ubicaron protecciones de sobre corriente para la bomba, la puesta a tierra del sistema y en el tablero principal una parada de emergencia.

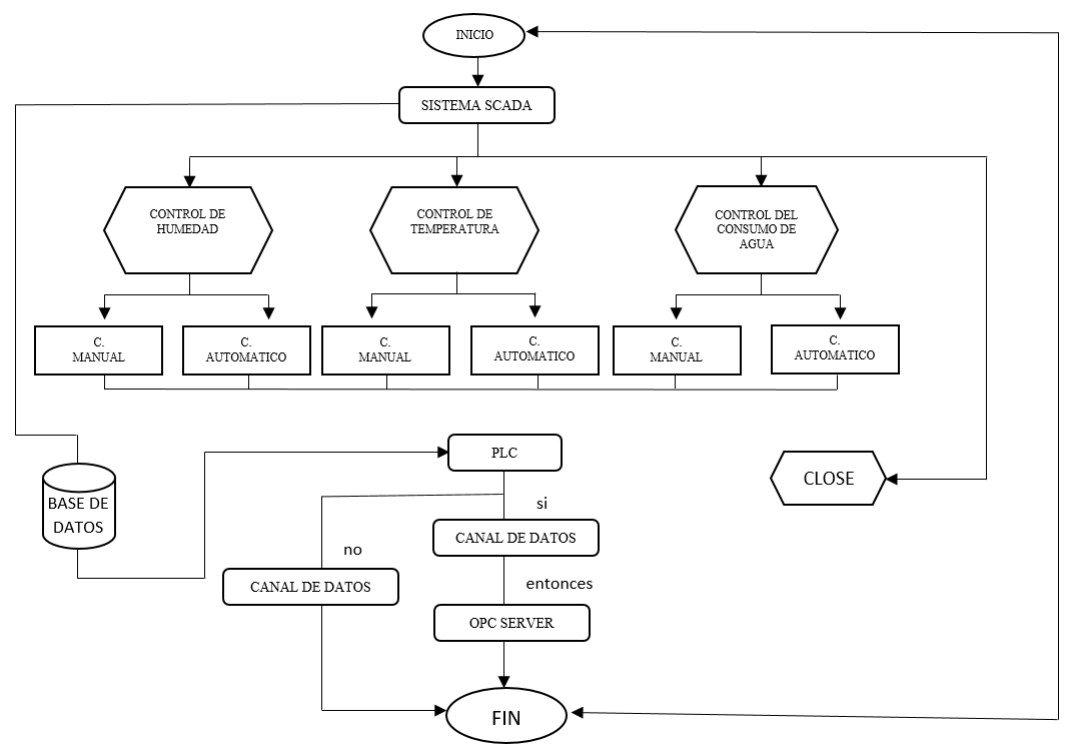

Fig. 11. Algoritmo general del sistema de control del invernadero 


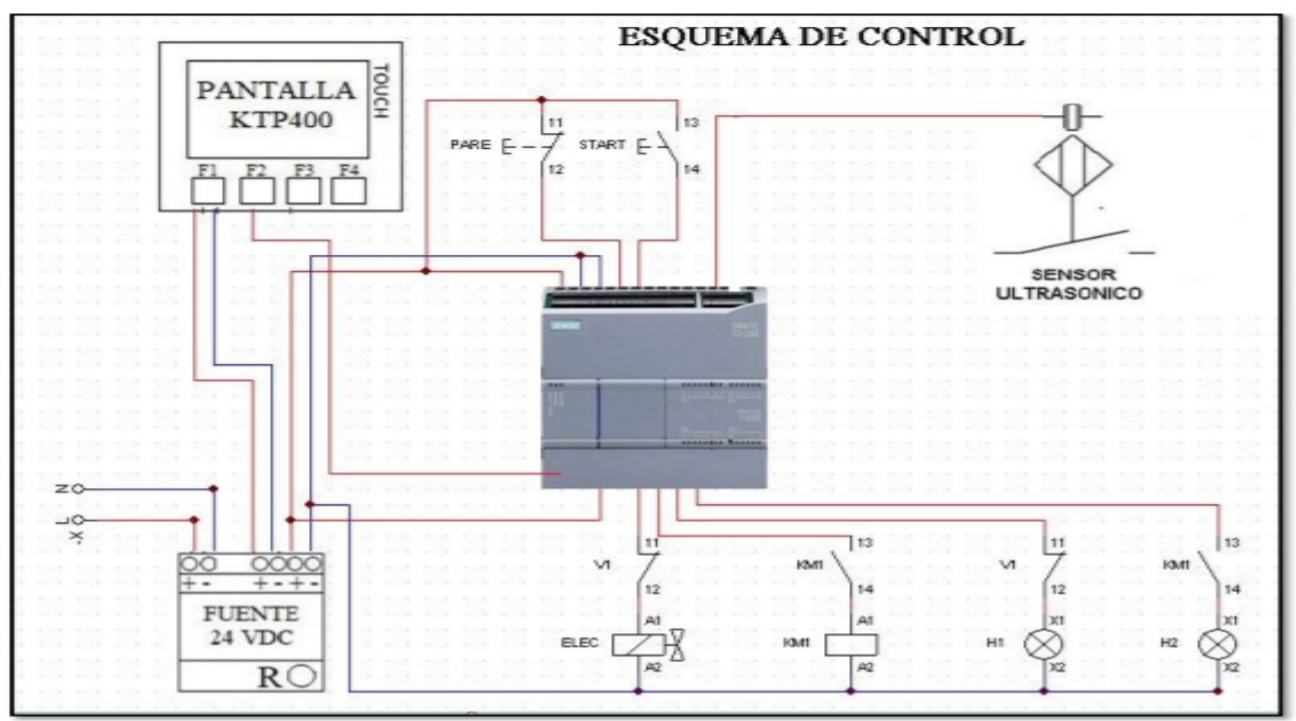

Fig. 12. Configuración del sistema de control del consumo de agua

El comportamiento de la humedad relativa obtenido en el invernadero se encuentra representado en la figura 13, que es obtenida con los registros de la base de datos del sistema, donde se utilizan datos en un tiempo de 20 minutos, durante
8 días con la programación del régimen de humedad y atendiendo a las necesidades de las plantas en presencia de perturbaciones como las variaciones de temperatura, radiación y humedad.

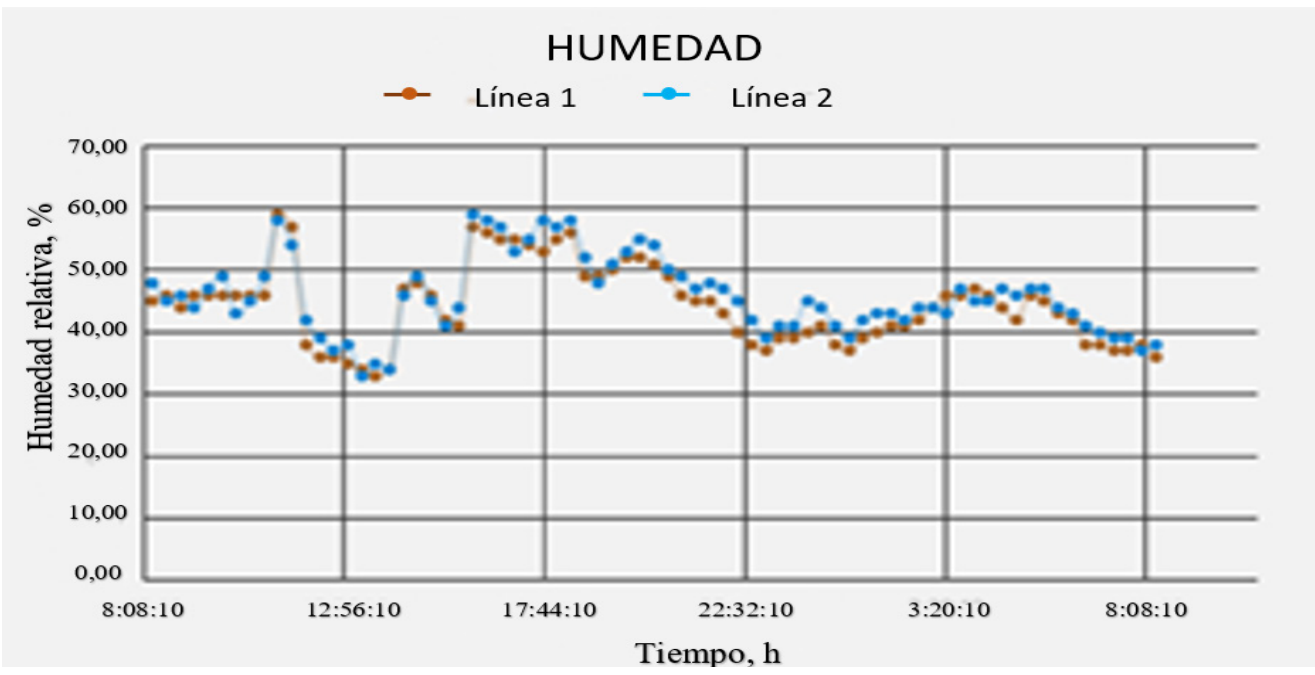

Fig. 13. Comportamiento de la humedad en el invernadero

Aquí se observa que es posible mantener el nivel de humedad por encima del $33 \%$, que resulta favorable para los cultivos. Con el control de los parámetros de explotación del invernadero, se pudo lograr un buen desarrollo de los cultivos, similar a los resultados de sistemas automatizados de [15] y [16].

En la figura 14 se muestra la pantalla que visualiza en el sistema los datos del consumo de agua, nivel y volumen del tanque, fecha, registro y consumo entre otros. Mientras que en la tabla II se relacionan la data que es almacenada en el servidor, donde se indica la capacidad máxima del tanque de 444 litros, los 318 litros consumidos, el nivel en el tanque de $20,7 \mathrm{~cm}$ y el volumen existente de 126 litros. Luego de realizar varios ensayos se pudo comprobar que existe un error promedio es de $0.2 \%$ en la medición de nivel. 


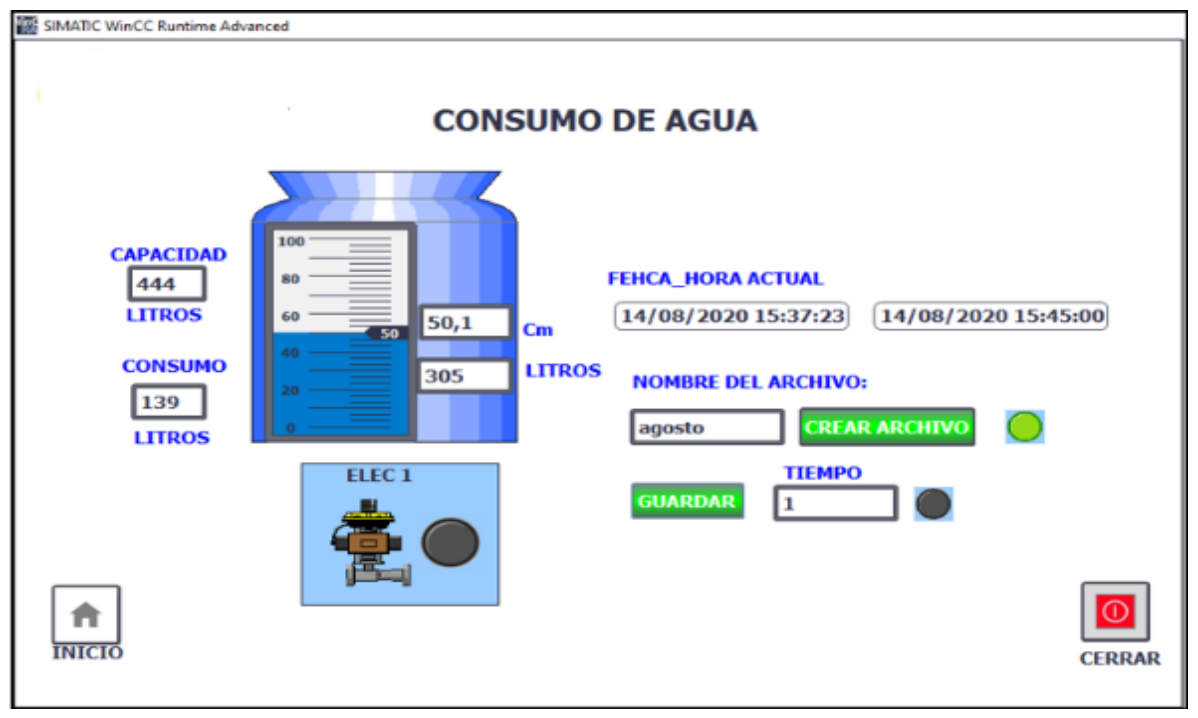

Fig. 14. Pantalla del consumo de agua para un ensayo

$\mathrm{Al}$ igual que en los trabajos [17] - [18] es posible obtener el uso eficiente del recurso hídrico, lo que incentiva la aplicación de esta tecnología para los sistemas de monitoreo y control en el sector agrícola. En este caso particular, donde el monitoreo y control de variables climatológicas del invernadero, incluye el manejo eficiente del recurso hídrico, y además la posibilidad de conocer el estado del sistema en todo momento, se garantiza una producción en ambiente controlado que asegura la explotación óptima del invernadero. Esto, pone de manifiesto la importancia del monitoreo y control para conocer el estado de las variables de operación, tanto del suelo como del ambiente, donde esta información le permitir al productor, el poder tomar acciones oportunas para el mejoramiento de la producción y la reducción de costos.

Tabla 2. Datos del registro del sistema durante los ensayos

Fecha Hora Nive1, Cm Volumen, L Disponibilidad (L) Consumo (L)

\begin{tabular}{l|lllll}
\hline $\mathbf{3} / \mathbf{0 8} / \mathbf{2 0 2 0}$ & $13: 12: 56$ & 0 & 444 & 0 & 444 \\
$\mathbf{3 / 0 8 / 2 0 2 0}$ & $13: 13: 56$ & 5.1 & 444 & 31 & 413 \\
$\mathbf{3 / 0 8 / 2 0 2 0}$ & $13: 14: 56$ & 5.8 & 444 & 35 & 409 \\
$\mathbf{3 / 0 8 / 2 0 2 0}$ & $13: 15: 56$ & 6.4 & 444 & 39 & 405 \\
$\mathbf{3 / 0 8 / 2 0 2 0}$ & $13: 16: 56$ & 6.9 & 444 & 42 & 402
\end{tabular}

\section{Conclusiones}

-El sistema para el control de humedad y nivel fue programado en el WinCC, lo que resultado ser amigable y garantizó los requerimientos climatológicos de los cultivos, además de ser compatible con la instrumentación de SIEMENS existente ya en el invernadero. Esto resultó una tecnología que garantiza la explotación óptima de invernadero y las condiciones climatológicas programadas por el operador para los cultivos.

-El control de humedad relativa tiene un comportamiento apropiado para los cultivos plantados al variar entre $33 \mathrm{y}$ $60 \%$ en el transcurso del día, según la programación y ello permitió el buen desarrollo del cultivo en un ambiente controlado, ya que se consideró favorable en las plantas, niveles de humedad superiores al $30 \%$ para garantizar una buena fotosíntesis y reducir la presencia de plagas.

-El monitoreo del consumo de agua se realizó con un medidor ultrasónico, que es un dispositivo sin contacto, con la medición continua y el error obtenido es inferior al $1 \%$, lo que permite accionar la electroválvula para poder entregar hasta $240 \mathrm{~L} / \mathrm{min}$. Además, es posible visualizar el nivel del tanque y el consumo de agua diario con la opción de tener reportes periodos del gasto de agua en el riego.

-En el HMI del control del consumo de agua se puede evidenciar las tres variables para activar una electroválvula, cuando el nivel del agua llegue al valor establecido por el operador, que este caso se ha seleccionado el volumen 150 
litros para tener más de un día de reserva en el riego. El sistema resultado ser amigable y garantizó los requerimientos climatológicos de los cultivos con una tecnología que garantiza la explotación óptima de invernadero y las condiciones climatológicas programadas.

-El control de humedad relativa tiene un comportamiento apropiado para los cultivos plantados al variar entre $33 \mathrm{y}$ $60 \%$ en el transcurso del día, según la programación y ello permitió el buen desarrollo del cultivo en un ambiente controlado, ya que se consideró favorable en las plantas, niveles de humedad superiores al $30 \%$ para garantizar una buena fotosíntesis y reducir la presencia de plagas.

-El monitoreo del consumo de agua se realizó con un instrumento ultrasónico, que es un medidor sin contacto, con la medición continua y el error obtenido es inferior al $1 \%$, que permite accionar la electroválvula para poder entregar hasta $240 \mathrm{~L} / \mathrm{min}$. Además, es posible visualizar el nivel del tanque y el consumo de agua diario con la opción de tener reportes periodos del gasto de agua en el riego.

-En el HMI del control del consumo de agua se puede evidenciar las tres variables para activar una electroválvula, cuando el nivel del agua llegue al valor establecido por el operador, que este caso se ha seleccionado el volumen 150 litros para tener más de un día de reserva en el riego.

\section{Referencias}

[1]M. Mamani, M. Villalobos y R. Herrera, «Sistema web de bajo costo para monitorear y controlar un invernadero agrícola,» Ingeniare Revista Chilena de Ingenieria, vol. $25, \mathrm{n}^{\circ} 4$, pp. 519-618, 2017.

[2]H. T. Tangarife, S. X. Toro y C. V. Carmona, «Sistemas automatizados para el control del recurso hídrico y variables ambientales bajo invernadero: aplicaciones y tendencias.,» Entre Ciencia e Ingeniería, vol. 14, nº 27, pp. 91-98, 2020.

[3]S. I. Ossa Duque, «Monitoreo y control de variables ambientales mediante una red inalámbrica para agricultura de precisión de invernaderos,» Vector $\mathrm{n}^{\circ}$ 12, pp. 51-60, 2017.

[4]I. L. López Cruz y L. Hernandez-Larragoti, «Modelos neuro-difusos para temperatura y humedad del aire en invernaderos tipo cenital y vapilla en el centro de Mexico,» Agrociencia, vol. 44, $\mathrm{n}^{\mathrm{o}}$ 7, pp. 791-805, 2010.

[5]D. M. Atia y T. El-madany, «Analysis and design of greenhouse temperature control using adaptive neuro-fuzzy inference system,» Journal of Electrical Systems and Information Technology, vol. 4, n 1, pp. 34-48, 2017.

[6]H. Burgueño Camacho, A. Reyes Rosas, R. Rodríguez García, A. Zermeño-González, D. Jasso Cantú y M. Cadena Zapata, «Evaluación de un modelo para estimar la temperatura y humedad relativa en el interior de invernadero con ventilación natural,» Chapingo Ser.Hortic, vol. 18, $\mathrm{n}^{\circ}$ 1, pp. 125-140, 2012.

[7]R. Salazar Moreno, A. M. Mauricio Pérez, I. L. López Cruz y A. Rojano Aguilar, «Un modelo de humedad dentro de un invernadero semicerrado,» Chapingo Ser.Hortic,vol. 22, $\mathrm{n}^{\mathrm{o}}$ 1, pp. 27-43, 2016.

[8]C. Sánchez Pérez, J. L. Guzmán, J. A. Sánchez Molina y
M. Berenguel, «Modelado y control multivariable de temperatura y humedad en un invernadero,» de VIII Congreso Ibérico de agroingeniería , Algorfa, España, 2015, pp. 856-865. [9]E. Alvarez Sánchez, G. Leyva Retureta, E. Portilla Flores y A. López Velázquez, «Evaluation of thermal behavior for an asymmetric greenhouse by means of dynamic simulations,» DYNA, vol. 81, n 188, pp. 151-159, 2014.

[10]R. Mena, J. A. Sánchez Molina, F. Rodríguez, L. L. Guzmán y M. Berenguel, «Diseño de un Sistema SCADA Modulable y Escalable para el Control de Clima y Riego en Invernaderos,» de VIII Congreso Ibérico de agroingeniería, España, 2015, pp. 946-957.

[11]L. Bárzaga Martell, R. C. Mompie Paneque y B. Valdés Cuesta, «Sistemas SCADA para la automatización de los procesos productivos del CIGB,» RIELAC, vol. 37, $\mathrm{n}^{\circ} 1$, pp. 20-37, 2016.

[12]A. Creus, Instrumentación Industrial, 8va. ed, Barcelona - España, Marcombo S.A, 2010, pp. 215-216.

[13]E. C. Martin y C. Muñoz, «Métodos para Medir la Humedad del Suelo para la Programación del Riego ¿Cuándo?,» University of Arizona, AZ1220s, Tucson, 2017.

[14]M. Cea y S. Alonso, «Sistema de Ayuda a la Decisión para la Programación del Riego Basado en Aspectos Agronómicos,» de IV Jornadas de Ingeniería del Agua, Córdoba, España, 2015, pp. 341-350.

[15]G. Quesada Roldán, «Producción de chile dulce en invernadero bajo diferentes niveles de agotamiento en la humedad del sustrato,» Agronomía Costarricense, vol. 39, n ${ }^{\circ}$ 1, pp. 25 36, 2015.

[16]A. Barroso García, «Control y monitorización de un invernadero a través de una aplicación móvil,» MSc. Tesis. Universidad Politécnica de Madrid, España, 2015.

[17]P. Muñoz, J. Buitrago, A. Arboleda, O. Cortes, A. Sánchez y C. Zapata, «Sistema de instrumentación y monitoreo para el invernadero la Aldana de la Universidad del Quindío,» Scientia et Technica, vol. 16, nº 49, pp. 219-225, 2011. [18]E. A. Villagrán, R. Gill, J. F. Acuña y C. R. Bojacá, «Optimization of ventilation and its effect on the microclimate of a colombian multispan greenhouse,» Agronomía Colombiana, vol. 30, no 2, pp. 282-288, 2012.

\section{RESUMEN CURRICULAR}

Marrero Ramírez Secundino: Graduado de Ing. Electrónico del Instituto Politécnico de Kiev y PhD en Automatización de la Universidad Minera de San Petersburgo, Rusia. Docente titular de la Universidad Técnica de Cotopaxi

González Palau Iliana: Ing. Electricista del Instituto de Minas de Moscú y PhD en Electricidad de la Universidad Minera de San Petersburgo, Rusia. Docente de la Universidad Técnica de Cotopaxi

León Segovia Marco Anibal: Graduado de Ing. En Eje- 
cución de Electrónica e instrumentación en la Escuela Politécnica del Ejercito, Latacunga Ecuador. MSc. Gestión de Energía y docente de la Universidad Técnica de Cotopaxi, Ecuador.
Suarez Vinueza Rommel Eusebio: Graduado de Ing. Electrónico en la Escuela Politécnica del Chimborazo, Riobamba Ecuador. MSc. en Gestión de Energía y docente de la Universidad Técnica de Cotopaxi, Latacunga Ecuador. 